\title{
ON THE THEOREMS OF ŠARKOVSKIĬ AND ŠTEFAN ON CYCLES
}

\author{
BOLESŁAW GAWE \\ (Communicated by R. Daniel Mauldin)
}

\begin{abstract}
New proofs of the well-known theorems of Šarkovskii and Štefan on cycles of a continuous real mapping are given.
\end{abstract}

Let us fix a real interval $I$ and a continuous function $f: I \rightarrow I$. For any $n \in \mathbf{N}$ we denote by $f^{n}$ the $n$-th iterate of $f$. A point $x \in I$ is said to be a periodic point of $f$ if $x$ is a fixed point of $f^{n}$ for some $n \in \mathbf{N}$. If $x$ is periodic, the smallest $n \in \mathbf{N}$ with $f^{n}(x)=x$ is called the period of $x$. Throughout this paper, $\operatorname{Per}(f, n)$ will stand for the set of all periodic points of $f$ of the period $n$.

Let us consider the following ordering of the set $\mathbf{N}$ :

$3 \dashv 5 \dashv 7 \dashv \cdots \dashv 2 \cdot 3 \dashv 2 \cdot 5 \dashv 2 \cdot 7 \dashv \cdots \dashv 2^{n} \cdot 3 \dashv 2^{n} \cdot 5 \dashv \cdots \dashv 2^{n} \dashv \cdots \dashv 2^{2} \dashv 2 \dashv 1$.

The aim of this paper is to give new proofs of the following two theorems.

Theorem A (A. N. Šarkovskiī). Let $n \in \mathbf{N}$. If $\operatorname{Per}(f, n) \neq \varnothing$ then $\operatorname{Per}(f, m) \neq$ $\varnothing$ for any $m \in \mathbf{N}$ such that $n \dashv m$.

Theorem B (P. Stefan). Let $n \geq 3$ be odd. Assume that $\operatorname{Per}(f, m)=\varnothing$ for any $m \dashv n$. If $x_{0} \in \operatorname{Per}(f, n)$ then there exists a point $x \in\left\{x_{0}, \ldots, f^{n-1}\left(x_{0}\right)\right\}$ such that

$$
x<f^{n-2}(x)<\cdots<f(x)<f^{2}(x)<\cdots<f^{n-1}(x)
$$

or

$$
x>f^{n-2}(x)>\cdots>f(x)>f^{2}(x)>\cdots>f^{n-1}(x) .
$$

Theorem A is a well-known result proved originally by A. N. Šarkovskii [5]. Another proof of Theorem A was given by P. Štefan in [6] who filled some gaps in Šarkovskiìs argument. Making use of some parts of his proof (cf. [6, Lemmas (20) and (21)]) Štefan proved Theorem B, which shows how $f$ acts on its minimal orbits of odd period. Chung-Wu Ho and Ch. Morris [4] also proved Theorems A and B by following some ideas of P. D. Straffin Jr. concerning directed graphs (cf. [7]). Another proof of Theorem A using the work of Straffin and digraphs was given by U. Burkart [3]. A sketch of a proof

Received by the editors March 24, 1988 and, in revised form, September 1, 1988.

1980 Mathematics Subject Classification (1985 Revision). Primary 26A18; Secondary 58F20. 
of Theorem of Šarkovskii may be found also in the paper [2]. Some comments concerning the history of the Šarkovskiì's result as well as the Burkart's proof have been presented with details by Gy. Targonski in his monograph $[8, \mathrm{Ch} .8$, $\S 2]$.

Up to now the greatest difficulty in proving Theorem $A$ has been to prove it in the particular case where $n$ is odd (Lemma 8 below). Usually it takes the largest parts of the proofs (cf. for example [5, Theorem 4 and Lemmas 5 and 6] or [6, (15), (16), (18) and Section D]). We proceed in a different, more natural way starting with a direct proof of Theorem B. Then we can simply prove Lemma 8 making use of the following three facts: Theorem B, Lemma 6 (which is actually a result of L. Block [1]), and a simple Lemma 7 . Consequently we obtain another proof of Theorem B and a new, rather short and clear proof of the Theorem of Šarkovskii. Lemmas 1, 2, 3, 4, 9, and 10 are standard and may be found for example in [6]. Nevertheless we prove them (Lemma 3 in a shorter way) for the convenience of the reader. Also Lemma 6 is presented with a short argument.

Lemma 1. Assume that $m, n \in \mathbf{N}$.

(i) $x \in \operatorname{Per}\left(f^{n}, 1\right)$ iff $x \in \operatorname{Per} f$ and the order of $x$ divides $n$.

(ii) $\operatorname{Per}(f, n) \subset \operatorname{Per}\left(f^{m}, n / k\right)$ where $k$ is the highest common divisor of $n$ and $m$.

(iii) If $p$ is prime then $\operatorname{Per}\left(f, p^{n+m}\right)=\operatorname{Per}\left(f^{p^{n}}, p^{m}\right)$.

Proof of Theorem $B$. If $n=3$, the statement is evident. Thus we can assume that $n \geq 5$. Let $C=\left\{x_{0}, \ldots, f^{n-1}\left(x_{0}\right)\right\}$. Since $n$ is odd, there exists a point $y \in C$ such that

$$
y<f(y)<f^{2}(y) \text { or } y>f(y)>f^{2}(y) .
$$

Assume for example the first of the above conditions. Put

$$
x=\min \left\{y \in C: y<f(y)<f^{2}(y)\right\} \text { and } y=f^{n-1}(x) .
$$

Clearly $x<f(x)<f^{2}(x)$ and $x<y$. We show that $f^{2}(x)<y$. Suppose on the contrary that $y \in(x, f(x)) \cup\left(f(x), f^{2}(x)\right)$. Consider the case $y \in$ $(x, f(x))$. Then

$$
f(y)=x<y<f(x),
$$

whence $\operatorname{Per}(f, 1) \cap(x, y) \neq \varnothing$. Let $a$ be its maximal point. Since

$$
f^{2}(a)=a<y<f(x)=f^{2}(y)
$$

there exists a $z_{1} \in(a, y)$ such that $f^{2}\left(z_{1}\right)=y$. Thus

$$
f^{3}\left(z_{1}\right)=x<z_{1} \text { and } y<f^{2}(x)=f^{3}(y)
$$

which (cf. the assumption of the theorem, Lemma 1(i), and the definition of $a$ ) is impossible. Now suppose that $y \in\left(f(x), f^{2}(x)\right)$. Put $a=\sup \operatorname{Per}(f, 1) \cap$ $(f(x), y)$. Since

$$
f(a)=a<y<f(f(x))
$$


there is a $z_{1} \in(f(x), a)$ such that $f\left(z_{1}\right)=y$. Due to the inequalities

$$
f(y)=x<z_{1}<a=f(a)
$$

we can find a $z_{2} \in(a, y)$ for which $f\left(z_{2}\right)=z_{1}$. Therefore we have

$$
f^{3}\left(z_{2}\right)=x<z_{2} \text { and } y<f^{2}(x)=f^{3}(y)
$$

which again leads to a contradiction. We now have that $f^{2}(x)<y$.

Let us observe that, by the inequalities

$$
f(x)<y=f^{n-2}(f(x)), \quad f^{n-2}\left(f^{2}(x)\right)=x<f^{2}(x),
$$

Lemma $1(\mathrm{i})$ and the assumption of the theorem, $\operatorname{Per}(f, 1) \cap\left(f(x), f^{2}(x)\right) \neq \varnothing$. Put

$$
a_{1}=\inf \operatorname{Per}(f, 1) \cap\left(f(x), f^{2}(x)\right)
$$

and

$$
a_{2}=\sup \operatorname{Per}(f, 1) \cap\left(f(x), f^{2}(x)\right) .
$$

Now, we will show, that the following condition is fulfilled for any $k \in$ $\{1, \ldots,(n-1) / 2\}$

$$
\text { if } \begin{gathered}
f^{2 k-1}(x)<\cdots<f(x)<f^{2}(x)<\cdots<f^{2 k}(x) \text {, then } \\
\left(\left(f^{2 k-1}(x), a_{1}\right) \cup\left(a_{2}, f^{2 k}(x)\right)\right) \cap \operatorname{Per}(f, 1)=\varnothing .
\end{gathered}
$$

For fix a $k \in\{1, \ldots,(n-1) / 2\}$ and suppose on the contrary that (1) does not hold. Clearly we may assume that $k \geq 2$. At first let $a$ be a point of $\operatorname{Per}(f, 1) \cap\left(f^{2 k-1}(x), f(x)\right)$. From the inequality

$$
f^{2 k-2}(f(x))=f^{2 k-1}(x)<a<a_{1}=f^{2 k-2}\left(a_{1}\right)
$$

we deduce, that $f^{2 k-2}\left(z_{1}\right)=a$ for a $z_{1} \in\left(f(x), a_{1}\right)$. Hence

$$
f(x)<f^{2 k}(x)=f^{2 k-1}(f(x))
$$

and

$$
f^{2 k-1}\left(z_{1}\right)=f\left(f^{2 k-2}\left(z_{1}\right)\right)=a<z_{1},
$$

which contradicts our assumptions. Now suppose that $\operatorname{Per}(f, 1) \cap\left(f^{2 i}(x), f^{2 i+2}\right.$ $(x)) \neq \varnothing$ for an $i \in\{1, \ldots, k-1\}$. Fix an element $a$ of this set. Since

$$
f^{2 i}\left(a_{2}\right)=a_{2}<a<f^{2 i+2}(x)=f^{2 i}\left(f^{2}(x)\right),
$$

there exists a $z_{1} \in\left(a_{2}, f^{2}(x)\right)$ such that $f^{2 i}\left(z_{1}\right)=a$ which, in view of the inequalities

$$
f^{n-2}\left(f^{2}(x)\right)=x<f^{2}(x) \text { and } z_{1}<a=f^{n-2 i-2}(a)=f^{n-2}\left(z_{1}\right),
$$

contradicts our assumptions and finishes the proof of (1).

To complete the proof of Theorem B it is enough to show inductively that, for any $k \in\{1, \ldots,(n-1) / 2\}$

$$
x<f^{2 k-1}(x)<\cdots<f(x)<f^{2}(x)<\cdots<f^{2 k}(x) \leq f^{n-1}(x)=y .
$$


If $k=1$ then (2) follows from the definition of $x$. Fix a $k \in\{1, \ldots,(n-3) / 2\}$ and assume (2). At first we shall show that $f^{2 k+1}(x) \in\left(x, f^{2 k-1}(x)\right)$. Suppose that $f^{2 k+1}(x)>f^{2 k}(x)$. Then for an $a \in \operatorname{Per}(f, 1) \cap\left(f^{2 k}(x), y\right)$,

$$
f^{n-3}\left(a_{2}\right)=a_{2}<a<y=f^{n-3}\left(f^{2}(x)\right)
$$

whence there exists a $z_{1} \in\left(a_{2}, f^{2}(x)\right)$ such that $f^{n-3}\left(z_{1}\right)=a$. Therefore

$$
f^{n-2}\left(f^{2}(x)\right)=x<f^{2}(x) \text { and } z_{1}<a=f(a)=f\left(f^{n-3}\left(z_{1}\right)\right)=f^{n-2}\left(z_{1}\right)
$$

which leads to a contradiction and proves that $f^{2 k+1}(x)<f^{2 k}(x)$. Now suppose that $f^{2 k+1}(x) \in\left(a_{1}, f^{2 k}(x)\right)$. From the inequalities

$$
f^{2 k-1}\left(a_{1}\right)=a_{1}<f^{2 k+1}(x)<f^{2 k}(x)=f^{2 k-1}(f(x))
$$

we deduce that there exists a $z_{1} \in\left(f(x), a_{1}\right)$ such that $f^{2 k-1}\left(z_{1}\right)=f^{2 k+1}(x)$. Since

$$
f^{n-2}\left(z_{1}\right)=f^{n-2 k-1}\left(f^{2 k-1}\left(z_{1}\right)\right)=f^{n-2 k-1}\left(f^{2 k+1}(x)\right)=x<z_{1}
$$

and

$$
f(x)<y=f^{n-2}(f(x)),
$$

there is a fixed point of $f^{n-2}$ in $\left(f(x), z_{1}\right)$ which is impossible. If $f^{2 k+1}(x) \in$ $\left(f^{2 k-1}(x), a_{1}\right)$ then by the inequalities

$$
f^{n-2}\left(f^{2 k+1}(x)\right)=f^{2 k-1}(x)<f^{2 k+1}(x)
$$

and

$$
f(x)<y=f^{n-2}(f(x)),
$$

the interval with the endpoints $f(x)$ and $f^{2 k+1}(x)$ would contain a fixed point of $f^{n-2}$ and this contradicts condition (1). At last suppose that $f^{2 k+1}(x)<x$. Putting $a=\min (\{f(x)\} \cup(\operatorname{Per}(f, 1) \cap(x, f(x))))$ we have

$$
f^{2}(a)<a_{1}<f^{2}(x),
$$

whence $f^{2}\left(z_{1}\right)=a_{1}$ for a $z_{1} \in(x, a)$. Thus

$$
f^{2 k+1}(x)<x \text { and } f^{2 k+1}\left(z_{1}\right)=a_{1}>z_{1},
$$

which is impossible. Consequently, $f^{2 k+1}(x) \in\left(x, f^{2 k-1}(x)\right)$.

Now we shall show that $f^{2 k+2}(x) \in\left(f^{2 k}(x), y\right]$. Clearly we may assume that $k \leq(n-5) / 2$. At first suppose that $y<f^{2 k+2}(x)$. In view of the inequalities

$$
f^{2 k+1}\left(a_{1}\right)=a_{1}<y<f^{2 k+2}(x)=f^{2 k+1}(f(x))
$$

there exists a $z_{1} \in\left(f(x), a_{1}\right)$ such that $f^{2 k+1}\left(z_{1}\right)=y$. Since

$$
f^{2}(f(x))<f(x)<z_{1}<a_{1}=f^{2}\left(a_{1}\right),
$$


$z_{1}=f^{2}\left(z_{2}\right)$ for a $z_{2} \in\left(f(x), a_{1}\right)$. Thus

$$
f^{2 k+3}\left(z_{1}\right)=f^{2}(y)=f(x)<z_{1}
$$

and

$$
z_{2}<y=f^{2 k+1}\left(z_{1}\right)=f^{2 k+3}\left(z_{2}\right),
$$

which is false and proves that $f^{2 k+2}(x) \leq y$. Suppose that $f^{2 k+2}(x)<f(x)$. Since

$$
f^{2}(f(x))<f(x)<a_{1}=f^{2}\left(a_{1}\right)
$$

there exists a $z_{1} \in\left(f(x), a_{1}\right)$ such that $f^{2}\left(z_{1}\right)=f(x)$. Then we have

$$
f^{2 k+1}(f(x))=f^{2 k+2}(x)<f(x)
$$

and

$$
z_{1}<f^{2 k}(x)=f^{2 k-1}(f(x))=f^{2 k+1}\left(z_{1}\right),
$$

which is impossible. Hence $f^{2 k+2}(x)>f(x)$. Now we shall prove that

$$
\left(f^{2 k+1}(x), f^{2 k-1}(x)\right) \cap \operatorname{Per}(f, 1)=\varnothing .
$$

Indeed, suppose that this is not the case and put $a=\min \operatorname{Per}(f, 1) \cap\left(f^{2 k+1}(x)\right.$, $\left.f^{2 k-1}(x)\right)$. Since

$$
f(a)=a<f^{2 k-1}(x) \leq f(x)<a_{2}<f^{2 k}(x)=f\left(f^{2 k-1}(x)\right),
$$

there exist a $z_{1} \in\left(a, f^{2 k-1}(x)\right)$ and $z_{2}, z_{3} \in\left(f^{2 k+1}(x), a\right)$ such that $f\left(z_{1}\right)=$ $a_{2}, f\left(z_{2}\right)=z_{1}, f\left(z_{3}\right)=f^{2 k-1}(x)$. Then

$$
f^{3}\left(z_{2}\right)=f^{2}\left(z_{1}\right)=a_{2}>z_{2}, \quad f^{3}\left(z_{3}\right)=f^{2}\left(f^{2 k-1}(x)\right)=f^{2 k+1}(x)<z_{3},
$$

contrary to the definition of $a$. This completes the proof of (3). Suppose that $f^{2 k+2}(x) \in\left(f(x), a_{2}\right)$. Then, since

$$
f\left(f^{2 k+1}(x)\right)<a_{2}<f(f(x)),
$$

we can find a $z_{1} \in\left(f^{2 k+1}(x), f(x)\right)$ with $f\left(z_{1}\right)=a_{2}$. Furthermore, by the inequalities

$$
f\left(f^{2}(x)\right)=f^{3}(x)<f(x)<f^{2 k+2}(x)<a_{2}=f\left(a_{2}\right),
$$

there exist a $z_{2} \in\left(a_{2}, f^{2}(x)\right)$ and a $z_{3} \in\left(f(x), a_{1}\right)$ such that $f\left(z_{2}\right)=f^{2 k+2}(x)$ and $f\left(z_{3}\right)=z_{2}$. Thus

$$
z_{1}<a_{2}=f^{n-2 k-1}\left(a_{2}\right)=f^{n-2 k-1}\left(f\left(z_{1}\right)\right)=f^{n-2 k}\left(z_{1}\right)
$$

and

$$
f^{n-2 k}\left(z_{3}\right)=f^{n-2 k-2}\left(f^{2}\left(z_{3}\right)\right)=f^{n-2 k-2}\left(f^{2 k+2}(x)\right)=x<z_{3},
$$

which contradicts conditions (1) and (3). At last if $f^{2 k+2}(x) \in\left(a_{2}, f^{2 k}(x)\right)$ then

$$
f^{n-2}\left(f^{2 k}(x)\right)=f^{2 k-2}(x)<f^{2 k}(x)
$$


and

$$
f^{2 k+2}(x)<f^{2 k}(x)=f^{n-2}\left(f^{2 k+2}(x)\right),
$$

contrary to (1). This completes the proof of Theorem B.

The proof of Theorem A consists of the following Lemmas 2-10.

Lemma 2. If $\operatorname{Per}(f, n) \neq \varnothing$ for some $n \in \mathbf{N}$ then $\operatorname{Per}(f, 1) \neq \varnothing$.

Lemma 3. If $\operatorname{Per}(f, n) \neq \varnothing$ for some $n \geq 2$ then $\operatorname{Per}(f, 2) \neq \varnothing$.

Proof. Fix an $x_{0} \in \operatorname{Per}(f, n)$ and put $x=\min \left\{x_{0}, \ldots, f^{n-1}\left(x_{0}\right)\right\}$. Let $y \in$ $\left\{x_{0}, \ldots, f^{n-1}\left(x_{0}\right)\right\}$ and $z \in[x, y)$ be such that $f(y)=x$ and $f(z)=y$. If $z=x$ then $x \in \operatorname{Per}(f, 2)$. Thus we may assume that $x<z$. Put

$$
u=\sup \left\{v \in[x, z]: f^{2}(v)=v\right\} \text {. }
$$

Then $u<z$. Suppose that $f(u)=u$ and choose an $a \in(z, y) \cap \operatorname{Per}(f, 1)$. Since

$$
f(u)=u<a<y=f(z)
$$

we have $a=f(b)$ for some $b \in(u, z)$. Thus $f^{2}(b)>b$ and $f^{2}(z)<z$ contrary to the definition of $u$. Consequently $u \in \operatorname{Per}(f, 2)$.

Lemma 4. Let $n \in \mathbf{N}$. If $\operatorname{Per}\left(f, 2^{n}\right) \neq \varnothing$ then $\operatorname{Per}\left(f, 2^{k}\right) \neq \varnothing$ for any $k \in$ $\{0, \ldots, n\}$.

Proof. It is enough to consider $k \in\{2, \ldots, n\}$ only. By Lemma 1 (iii) $\operatorname{Per}\left(f^{2^{k-1}}\right.$, $\left.2^{n-k+1}\right) \neq \varnothing$. Using Lemma 3 and Lemma 1 (iii) again we have

$$
\varnothing \neq \operatorname{Per}\left(f^{2^{k-1}}, 2\right)=\operatorname{Per}\left(f, 2^{k}\right) .
$$

Lemma 5. Let $k \in \mathbf{N}_{0}$ and let $l \geq 3$ be odd. If $\operatorname{Per}\left(f, 2^{k} \cdot l\right) \neq \varnothing$ then $\operatorname{Per}\left(f, 2^{n}\right) \neq \varnothing$ for any $n \in \mathbf{N}_{0}$.

Proof. Use Lemma 1(ii) (taking $n=2^{k} l, m=2^{n+k}$ ), Lemma 3, and then Lemmas 1 (iii) and 4.

Lemma 6. If

$$
\begin{array}{ll}
f^{2 k}\left(x_{0}\right)<\cdots<f^{2}\left(x_{0}\right)<x_{0}<f\left(x_{0}\right)<\cdots<f^{2 k-1}\left(x_{0}\right), & f^{2 k+1}\left(x_{0}\right) \leq x_{0} \\
\text { or } \quad f^{2 k}\left(x_{0}\right)>\cdots>f^{2}\left(x_{0}\right)>x_{0}>f\left(x_{0}\right)>\cdots>f^{2 k-1}\left(x_{0}\right), & f^{2 k+1}\left(x_{0}\right) \geq x_{0}
\end{array}
$$

for some $x_{0} \in I$ and $k \in \mathbf{N}$ then $\operatorname{Per}(f, 2 k+1) \neq \varnothing$.

Proof. Let us assume that the first of the above conditions holds and fix an $a \in\left(x_{0}, f\left(x_{0}\right)\right) \cap \operatorname{Per}(f, 1)$. Let $I_{0}, \ldots, I_{2 k+1}$ be compact intervals such that

$$
I_{2 k+1}=\left[x_{0}, f^{2 k-1}\left(x_{0}\right)\right] \text {, }
$$

$I_{i}$ is contained in the compact interval with the endpoints

$$
\begin{aligned}
& f^{i}\left(x_{0}\right) \text { and } f^{i-2}\left(x_{0}\right), \quad i=2 k, \ldots, 2, \\
& I_{1} \subset\left[a, f\left(x_{0}\right)\right], \quad I_{0} \subset\left[x_{0}, a\right], \\
& f\left(I_{i}\right)=I_{i+1}, \quad i=2 k, \ldots, 0 .
\end{aligned}
$$


Then $f^{2 k+1}\left(I_{0}\right)=I_{2 k+1} \supset I_{0}$ whence we can find a $c \in I_{0} \cap \operatorname{Per}\left(f^{2 k+1}, 1\right)$. Thus it follows from the relations

$$
f^{i}\left(I_{0}\right) \cap I_{0} \subset\left\{x_{0}\right\}, \quad i=2, \ldots, 2 k \text { and } f\left(I_{0}\right) \cap I_{0} \subset\{a\}
$$

that $c \in \operatorname{Per}(f, 2 k+1)$.

Lemma 7. Assume that there exists an $x_{0} \in I$ such that

$$
f^{2}\left(x_{0}\right)<x_{0}<f\left(x_{0}\right) \text { and } f^{3}\left(x_{0}\right) \leq a
$$

or

$$
f^{2}\left(x_{0}\right)>x_{0}>f\left(x_{0}\right) \text { and } f^{3}\left(x_{0}\right) \geq a
$$

where $a \in \operatorname{Per}(f, 1)$ belongs to the interval with the endpoints $x_{0}$ and $f\left(x_{0}\right)$. Then, for any $k \in \mathbf{N}, \operatorname{Per}(f, 2 k) \neq \varnothing$.

Proof. Assume the first of the above cases. Take $x_{-1}=f\left(x_{0}\right)$. Since

$$
f\left(f\left(x_{0}\right)\right)<x_{0}<a=f(a),
$$

there is an $x_{1} \in\left(a, f\left(x_{0}\right)\right)$ such that $f\left(x_{1}\right)=x_{0}$. By the inequalities

$$
f(a)=a<x_{1}<f\left(x_{0}\right)
$$

we can find an $x_{2} \in\left(x_{0}, a\right)$ with $f\left(x_{2}\right)=x_{1}$. Continuing this procedure inductively we obtain a sequence $\left(x_{n}: n \in \mathbf{N}\right)$ with the properties

$$
x_{2 n-2}<x_{2 n}<a<x_{2 n-1}<x_{2 n-3} \text { and } f\left(x_{n}\right)=x_{n-1}, \quad n \in \mathbf{N} .
$$

Let $I_{0}, \ldots, I_{2 k}$ be compact intervals such that

$$
I_{2 k}=\left[f^{3}\left(x_{0}\right), f\left(x_{0}\right)\right], \quad I_{2 k-1} \subset\left[f^{2}\left(x_{0}\right), x_{0}\right],
$$

$I_{i}$ is contained in the compact interval with the endpoints

$$
f\left(I_{i}\right)=I_{i+1}, \quad i=2 k-1, \ldots, 0 . \quad \text { and } x_{2 k-i-3}, \quad i=2 k-2, \ldots, 0,
$$

Then $f^{2 k}\left(I_{0}\right)=I_{2 k} \supset I_{0}$ whence there is a $c \in I_{0} \cap \operatorname{Per}\left(f^{2 k}, 1\right)$. Thus, due to the relations

$$
f^{i}\left(I_{0}\right) \cap I_{0}=I_{i} \cap I_{0}=\varnothing, \quad i \in\{1, \ldots, 2 k-1\} \backslash\{2\},
$$

and (in the case $k>1$ )

$$
f^{2}\left(I_{0}\right) \cap I_{0}=I_{2} \cap I_{0}=\left\{x_{2 k-3}\right\},
$$

$c \in \operatorname{Per}(f, 2 k)$ which was to be proved.

Lemma 8. Let $n \geq 3$ be odd. If $\operatorname{Per}(f, n) \neq \varnothing$ then $\operatorname{Per}(f, m) \neq \varnothing$ for any $m \in \mathbf{N}$ such that $n \dashv m$.

Proof. It is enough to consider the case where $\operatorname{Per}(f, k)=\varnothing$ for any $k \dashv n$. In view of Theorem B we may assume for example that

$$
f^{n-1}\left(y_{0}\right)<\cdots<y_{0}<f\left(y_{0}\right)<\cdots<f^{n-2}\left(y_{0}\right)
$$


for a $y_{0} \in \operatorname{Per}(f, n)$. If $m$ is even then, taking $x_{0}=f^{n-3}\left(y_{0}\right)$ in Lemma 7, we get the assertion. Assume that $m>n$ is odd. Proceeding analogously as in the proof of Lemma 7 we get the sequence $\left(y_{i}: i=1, \ldots, m-n\right)$ such that

$$
y_{0}<y_{2}<\cdots<y_{m-n}<y_{m-n-1}<\cdots<y_{1}<f\left(y_{0}\right)
$$

and

$$
f\left(y_{i}\right)=y_{i-1}, \quad i=1, \ldots, m-n .
$$

Now it is enough to put $x_{0}=y_{m-n}$ and use Lemma 6 .

Lemma 9. Let $n \geq 2$. If $\operatorname{Per}\left(f^{2}, n\right) \neq \varnothing$ then $\operatorname{Per}(f, 2 n) \neq \varnothing$.

Proof. Let $x_{0} \in \operatorname{Per}\left(f^{2}, n\right)$ and let $r=\operatorname{card}\left\{x_{0}, \ldots, f^{2 n-1}\left(x_{0}\right)\right\}$. Clearly $r \geq n$ and $r \mid 2 n$. Thus, $r \in\{n, 2 n\}$. If $n$ is even then $f^{n}\left(x_{0}\right) \neq x_{0}$ whence $r=2 n$ and $x_{0} \in \operatorname{Per}(f, 2 n)$. If $n$ is odd and $r=n$ we may use Lemma 8 .

Lemma 10. Let $n \in \mathbf{N}_{0}$ and let $l \geq 3$ be odd. If $\operatorname{Per}\left(f, 2^{n} l\right) \neq \varnothing$ then $\operatorname{Per}\left(f, 2^{m} k\right) \neq \varnothing$ for $m=n$ and any odd $k \geq l$ and for any $m>n$ and odd $k \geq 3$.

Proof. If $n=0$ the assertion follows from Lemma 8. Fix an $n \in \mathbf{N}$ and assume that the lemma holds for $n-1$. By Lemma 1(ii) $\operatorname{Per}\left(f^{2}, 2^{n-1} l\right) \neq \varnothing$. Hence, in view of the induction hypothesis,

$$
\operatorname{Per}\left(f^{2}, 2^{m-1} k\right) \neq \varnothing, \quad m>n \text { and } k \geq 3 \text { or } m=n \text { and } k \geq 1 .
$$

Now use Lemma 9.

\section{REFERENCES}

1. L. Block, Stability of periodic orbits in the theorem of Sarkovskii, Proc. Amer. Math. Soc. 81 (1981), 333-336.

2. L. Block, J. Guckenheimer, M. Misiurewicz and L. S. Young, Periodic points and topological entropy, L.ecture Notes in Mathematics Nr. 819, Springer, Berlin (1980), 18-34.

3. U. Burkart, Interval mapping graphs and periodic points of continuous functions, Journ. of Comb. Theory 32 (1982), 57-68.

4. C. Ho and Ch. Morris, A graph-theoretic proof of Sharkovsky's theorem on the periodic points of continuous functions, Pacific J. Math. 96 (1981), 361-370.

5. A. N. Šarkovskiī, Coexistence of cycles of a continuous transformation of line into itself, Ukrain. Mat. Ż. 16 (1964), 61-71.

6. P. Štefan, A theorem of Sarkovskii on the existence of periodic orbits of continuous endomorphisms of the real line, Comun. Math. Phys. 54 (1977), 237-258.

7. P. D. Straffin, Jr., Periodic points of continuous functions, Math. Mag. 51 (1978), 99-105.

8. Gy. Targonski, Topics in iteration theory, Vandenhoeck und Ruprecht, 1981.

Department of Mathematics, Silesian University, Bankowa 14, 40-007 Katowice, POLAND 